This item was submitted to Loughborough's Research Repository by the author.

Items in Figshare are protected by copyright, with all rights reserved, unless otherwise indicated.

\title{
Wave group dynamics in weakly nonlinear long-wave models
}

PLEASE CITE THE PUBLISHED VERSION

LICENCE

CC BY-NC-ND 4.0

\section{REPOSITORY RECORD}

Grimshaw, Roger H.J., Dmitry Pelinovsky, Efim N. Pelinovsky, and Tatiana G. Talipova. 2019. "Wave Group Dynamics in Weakly Nonlinear Long-wave Models". figshare. https://hdl.handle.net/2134/767. 


\title{
Wave group dynamics in weakly nonlinear long-wave models
}

\author{
Roger Grimshaw \\ Department of Mathematical Sciences, Loughborough University, \\ Loughborough, UK \\ Dmitry Pelinovsky \\ Department of Mathematics, McMaster University, Hamilton, Canada \\ Efim Pelinovsky and Tatiana Talipova \\ Laboratory of Hydrophysics and Nonlinear Acoustics, \\ Institute of Applied Physics, Nizhny Novgorod, Russia
}

September 20, 2000

\begin{abstract}
Wave group dynamics is studied in the framework of the extended Korteweg-de Vries equation. The nonlinear Schrodinger equation is derived for weakly nonlinear wave packets, and the condition for modulational instability is obtained. It is shown that wave packets are unstable only for a positive sign of the coefficient of the cubic nonlinear term in the extended Korteweg-de Vries equation, and for a high carrier frequency. At the boundary of this parameter space, a modified nonlinear Schrodinger equation is derived, and its steady-state solutions, including an algebraic soliton, are found. The exact breather solution of the extended Korteweg-de Vries equation is analyzed. It is shown that in the limit of weak nonlinearity it transforms to a wave group with an envelope described by soliton solutions of the nonlinear Schrodinger equation and its modification as described above. Numerical simulations demonstrate the main features of wave group evolution and show some differences in the behavior of the solutions of the extended Korteweg-de Vries equation, compared with those of the nonlinear Schrodinger equation.
\end{abstract}

\section{Introduction}

Long-wave weakly nonlinear models are very popular in many physical fields, notably for the dynamics of stratified flows in the ocean and atmosphere. They have been derived for various environmental conditions of the density and shear flow stratification. The famous Korteweg-de Vries equation is the basic model obtained at the leading order of a perturbation theory based on the small parameters of nonlinearity (amplitude is less than a characteristic depth scale) and dispersion (wavelength is more than this depth scale). For certain environmental conditions, the coefficient of the quadratic nonlinear term is close to 
zero (for instance, when the pycnocline lies at the middle depth in a two-layer model of density stratification). In such cases the cubic nonlinear term in the second order of perturbation theory should be included, so that the extended Korteweg-de Vries equation is obtained. It contains both quadratic and cubic nonlinearity, and is given by

$$
\frac{\partial u}{\partial t}+\mu u \frac{\partial u}{\partial x}+\nu u^{2} \frac{\partial u}{\partial x}+\lambda \frac{\partial^{3} u}{\partial x^{3}}=0,
$$

where $u(x, t)$ is the amplitude of the relevant wave mode (e.g. $u$ may be the vertical displacement of pycnocline), $x$ is a horizontal coordinate and $t$ is time. The coefficients of this equation are obtained from the environmental density and shear flow stratification. It is important to note that the dispersion coefficient $\lambda$ is always positive, but that the nonlinear coefficients, $\mu$ and $\nu$, can have either sign. The review paper by Grimshaw [5] discusses the derivation of the extended Korteweg-de Vries equation (1) from the governing equations of the fluid motion, and also gives the expressions for all these coefficients.

The extended Korteweg-de Vries equation is a fully integrable model. A simple shift in the wave variable $u$ transforms it to the modified Korteweg-de Vries equation

$$
\frac{\partial v}{\partial t}+\nu v^{2} \frac{\partial v}{\partial y}+\lambda \frac{\partial^{3} v}{\partial y^{3}}=0,
$$

where $v=u+\mu / 2 \nu, y=x+\mu^{2} t / 2 \nu^{2}$. Its dynamics is determined by the sign of $\nu$ (assuming that $\lambda>0$ ). If $\nu<0$, the modified Korteweg-de Vries equation can be reduced to the Korteweg-de Vries equation through the Miura transformation [11]. No soliton solution (vanishing at infinity) exists in this case, but they can exist on a pedestal. Taking into account that the transformation from (1) to (2) also contains a pedestal, soliton solutions which are bounded at infinity, exist in the framework of the extended Korteweg-de Vries equation. The polarity of this soliton is determined by the sign of the coefficient $\mu$ of the quadratic nonlinear term. The amplitude is bounded by $A_{\mathrm{lim}}=-\mu / \nu$ and in this limiting case, the soliton becomes "thick" i.e. its width tends to infinity. Recently, the interaction of "thick" and "thin" solitons was studied by Slunyaev \& Pelinovsky [18]; a small soliton moving through a soliton of limiting amplitude changes its polarity.

If $\nu>0$, the wave dynamics is completely different. In particular, the soliton of the extended Korteweg-de Vries equation can have either polarity, but the soliton amplitude whose polarity is opposite in sign to the coefficient $\mu$ of the quadratic nonlinear term, should exceed (in modulus) the minimal value $A_{a l}=$ $-2 \mu / \nu$. The last case corresponds to an algebraic standing soliton. Additionally, the extended Korteweg-de Vries equation, with a positive coefficient $\nu$ of the cubic nonlinear term, has solutions in the form of oscillating packets (breathers), whose analytical expressions were found by Pelinovsky \& Grimshaw [14]. They showed also that the algebraic soliton is unstable transforming into a breather due to structural instability. The generation of solitons and breathers from an initial disturbance for the case $\mu=0$ recently was studied by Clarke et al., [3]. 
If the extended Korteweg-de Vries equation is weakly perturbed (for instance, through variable coefficients), the soliton can transform into a breather and vice versa [7].

The dynamics of quasi-periodic disturbances in the framework of these long wave models is much less well understood. First we note the work by Zakharov \& Kuznetsov [20] who obtained the nonlinear Schrodinger equation from the Korteweg-de Vries equation for weakly nonlinear wave packets. They showed that a wave group should demodulate. Numerical simulation of wind wave groups in shallow water, based on the Korteweg-de Vries equation, demonstrate the demodulation of wave groups for various amplitudes [19], [17]. It was shown that the envelope of a wave packet of moderate amplitudes varies significantly, becomes asymmetric and the wave packet splits into several groups with different carrier frequencies. Interesting examples of nonlinear wave focusing in shallow water are presented by Pelinovsky et al., [15] with application to the "freak" wave phenomenon. The nonlinear Schrodinger equation was also derived from the extended Korteweg-de Vries equation by Parkes [12], but the dynamics of the wave packets in this case was not investigated.

The aim of this paper is to study the dynamics of modulated wave groups in the framework of the extended Korteweg-de Vries equation. Both signs of the coefficient $\nu$ of the cubic nonlinear term are taken into account. For the limiting case of weakly nonlinear waves a nonlinear Schrodinger equation for the wave envelope is derived from the extended Korteweg-de Vries equation (section 2). The condition for modulational instability is found, and the wave group is unstable only when the coefficient of the cubic nonlinear term, $\nu$, is positive and the carrier wavenumber is large. If the wavenumber is less than $k_{c r}$ $\left(k_{c r}=\sqrt{\mu^{2} / 6 \lambda \nu}\right)$ the wave packet is stable. A modified Schrodinger equation is derived for wave parameters at the boundary of this parameter space (section 3). Steady-state solutions of this equation describing envelope solitons are found. It is shown that if the carrier wavenumber is less than $k_{c r}$ the soliton exists only when its amplitude exceeds the minimal value corresponing to the algebraic soliton. The exact breather solution of the extended Korteweg-de Vries equation is analysed in the weakly nonlinear limit (section 4). It is shown that this breather describes a wave group with an envelope of the form of the soliton of the modified nonlinear Schrodinger equation. Numerical simulations of the extended Korteweg-de Vries equation and the nonlinear Schrodinger equation are described in section 5. These simulations demonstrate the main features of wave group dynamics: demodulation of the wave packet, or its self modulation with formation of envelope solitons and breathers. The dynamics of wave groups within the extended Korteweg-de Vries equation is "richer" than that predicted by the nonlinear Schrodinger equation, in particular, wave packets develop asymmetry of the wave envelope during the evolution process. Further, wave packets may separate out. 


\section{Dynamics of weakly nonlinear wave packets}

First, the dynamics of very weak disturbances should be considered. In this case, the nonlinear terms in equation (1) are of less importance than the dispersive term. Such a situation typically produces the nonlinear Schrodinger equation for the envelope of quasi-sinusoidal waves. The applicability of the nonlinear Schrodinger equation in this context is well known (see, for instance, Ablowitz \& Segur [1]). In particular, the link between the classical Korteweg-de Vries equation, as well as the extended Korteweg-de Vries equation, and the nonlinear Schrodinger equation was studied by Zakharov \& Kuznetsov [20] and Parkes [12] respectively. Here we derive the nonlinear Schrodinger equation from the extended Korteweg-de Vries equation (1) using a slightly different asymptotic procedure to that of [12].

A solution of the extended Korteweg-de Vries equation is sought in the form

$$
u(x, t)=\epsilon A(X, T) \exp (i \Theta)+\epsilon^{2} A_{2}(X, T) \exp (2 i \Theta)+c . c+\epsilon^{2} A_{0}(X, T)+\ldots
$$

where $\Theta=k x-\omega t, \omega=-\lambda k^{3}, X=\epsilon x, T=\epsilon t, \epsilon<<1$ and c.c. is the complex conjugate. After substitution of (3) into (1), we obtain a set of equations corresponded to the different carrier harmonics. For the first harmonic it is

$$
\epsilon^{2}\left(\frac{\partial A}{\partial T}+c_{g} \frac{\partial A}{\partial X}\right)+\epsilon^{3}\left(i 3 \lambda k \frac{\partial^{2} A}{\partial X^{2}}+i \mu k\left(A_{2} A^{*}+A_{0} A\right)+i \nu k|A|^{2} A\right)+\ldots=0
$$

where $c_{g}=-3 \lambda k^{2}$ is the group velocity of the basic wave. Introducing the system of coordinates moving with group velocty, $\xi=X-c_{g} T$ and $\tau=\epsilon T$, equation (4) becomes, to an accuracy of $0\left(\epsilon^{4}\right)$ as

$$
\frac{\partial A}{\partial \tau}+i 3 \lambda k \frac{\partial^{2} A}{\partial \xi^{2}}+i k \mu\left(A_{2} A^{*}+A_{0} A\right)+i k \nu|A|^{2} A=0 .
$$

For the second harmonic we obtain

$$
\epsilon^{2}\left(\left(-2 i \omega-8 i \lambda k^{3}\right) A_{2}+i k \mu A^{2}\right)+\ldots=0,
$$

and with the same accuracy

$$
A_{2}=\frac{\mu}{6 \lambda k^{2}} A^{2}
$$

For the mean wave component similarly obtain

$$
\epsilon^{3}\left(\frac{\partial A_{0}}{\partial T}+\mu \frac{\partial|A|^{2}}{\partial X}\right)+\ldots=0 .
$$

Taking into account that $\partial / \partial T=-c_{g} \partial / \partial X$ is at the leading order, equation (8) may be solved with the same accuracy to give

$$
A_{0}=\frac{\mu}{c_{g}}|A|^{2}=-\frac{\mu}{3 \lambda k^{2}}|A|^{2}
$$


Consequently, equation (5) becomes the classical nonlinear Schrodinger equation,

$$
i \frac{\partial A}{\partial \tau}=3 \lambda k \frac{\partial^{2} A}{\partial \xi^{2}}+k \delta|A|^{2} A,
$$

where

$$
\delta=\nu-\frac{\mu^{2}}{6 \lambda k^{2}} .
$$

The nonlinear Schrodinger equation is a well-known model for the evolution of weakly nonlinear quasi-harmonic wave packet. The character of the wave dynamics, as is well known, depends on the sign of the Lighthill parameter,

$$
L=3 \lambda \delta k ;
$$

If it is negative, the wave packet is stable and demodulates; if it is positive, a plane wave is unstable and modulational instability (the Benjamin-Feir instability) develops. For surface and internal waves $\lambda>0$, and the sign of the Lighthill parameter depends on the sign of nonlinear coefficients. In particular, the classic Korteweg-de Vries equation with no cubic nonlinear term $(\nu=0)$ has no modulational instability for any sign of $\mu$ [20]. The same result can be obtained for shallow water waves from the full hydrodynamic equations, see for instance, [1]. A negative coefficient of the cubic nonlinear term $(\nu<0)$ leads to negative values of the nonlinear coefficient $\delta$ in $(10)$ and waves are stable. The coefficient, $\nu$ is negative for internal waves in a two-layer fluid [9], and, thus weakly nonlinear interfacial waves are always stable. A positive sign of the coefficient $\nu$ may appear for internal waves in the three-layer fluid [6], and the modulational instability is possible when

$$
k>\frac{|\mu|}{\sqrt{6 \nu \lambda}} .
$$

In particular, when equation (1) reduces to the modified Korteweg-de Vries equation $(\mu=0)$, the stability criterion does not contain the wavenumber, and plane waves of any carrier wavelength are stable if $\nu<0$ and unstable, if $\nu>0$. When the coefficient of the quadratic nonlinear term differs from zero, modulational instability exists only for high-frequency wave packets, see (13).

It is convenient to introduce new variables,

$$
A_{1}=\sqrt{\frac{|\delta|}{6|\lambda|}} A, \quad \eta=-3 \lambda k \tau,
$$

as that the nonlinear Schrodinger equation (10) reduces to the canonical form

$$
i \frac{\partial A_{1}}{\partial \eta}+\frac{\partial^{2} A_{1}}{\partial \xi^{2}} \pm 2\left|A_{1}\right|^{2} A_{1}=0 .
$$

where the sign in front of the nonlinear cubic term coincides with the sign of $\delta \lambda$. 
The case of a positive nonlinear term in equation (15) is the more interesting from a physical point of view due to the modulational instability mentioned above. The characteristic scales of this instability are the width $\Delta K$ of the unstable domain in the envelope wavenumber space, the optimal wavenumber $K$ and the maximum value $\Psi$ for increment of the Benjamin-Feir instability, given by

$$
\Delta K=2 A_{10}, \quad K=\sqrt{2} A_{10}, \quad \Psi=2 A_{10}^{2},
$$

where $A_{10}$ is the amplitude of plane unperturbed wave(see, e.g. 14). Also, we give here the expression for the envelope soliton

$$
A_{1}(\xi, \eta)=D \operatorname{sech}(D \xi)
$$

with arbitrary amplitude, $D$. It is important to mention that on the boundary in parameter space of the modulational instability, $\delta \rightarrow 0$, the soliton amplitude in the physical variables tends to the infinity as $\delta^{-1 / 2}$, see (14), and the wave group collapses.

The nonlinear Schrodinger equation may be solved exactly using the inverse scattering method [1], [11]. We give here only a few important exact solutions; these are the rational breather [16],

$$
A_{1}(\xi, \eta)=\left(1-\frac{4(1+4 i \eta)}{1+4 \xi^{2}+16 \eta^{2}}\right) \exp (2 i \eta),
$$

the time periodical breather [10],

$$
A_{1}(\xi, \eta)=\frac{\cos (\Omega \eta-2 i \varphi)-\cosh (\varphi) \cosh (p \xi)}{\cos (\Omega \eta)-\cosh (\varphi) \cosh (p \xi)} \exp (2 i \eta)
$$

where $p=2 \sinh (\varphi), \Omega=2 \sinh (2 \varphi)$ and $\varphi$ is free real parameter, and the space periodical breather [2],

$$
A_{1}(\xi, \eta)=\frac{\cosh (\Omega \eta-2 i \varphi)-\cos (\varphi) \cos (p \xi)}{\cosh (\Omega \eta)-\cos (\varphi) \cos (p \xi)} \exp (2 i \eta),
$$

where $p=2 \sin (\varphi), \Omega=2 \sin (2 \varphi)$ and $\varphi$ is again a free real parameter. In fact, (19) and (20) are equivalent and are special cases of a more general solution in which $\varphi$ is allowed to be complex in either expression, while the rational breather can be obtained in the limit $\varphi \rightarrow 0$. The breather solutions of the nonlinear Schrodinger equation play an important role in understanding the nonlinear origin of the "freak" wave phenomenon on the sea surface where the wave amplitude can be amplified up to three times [4], [8]. All these solutions can be used to test numerical solutions of the extended Korteweg-de Vries equation.

\section{Modification of the nonlinear Schrodinger equ- ation in the transition zone}

The case when the coefficient $\delta$ of the cubic nonlinear term in (10) is close to zero, i.e. $k \approx|\mu| / \sqrt{6 \nu \lambda}$, needs special consideration. A general approach to 
obtain a modified nonlinear Schrodinger equation is described in [12]. Here we use a slightly different asymptotic method to produce the modified nonlinear Schrodinger equation and obtain its solutions. When we continue the series (3) up to $0\left(\epsilon^{3}\right)$, including the third harmonic terms, introduce to re-scaled coordinates, $\xi=\epsilon\left(X-c_{g} T\right), \tau=\epsilon^{3} T$, and replace $\delta$ with $\epsilon^{2} \delta$, then equation (4) for amplitude of the first harmonic transforms to

$$
\begin{gathered}
\frac{\partial A}{\partial \tau}+3 i \lambda k \frac{\partial^{2} A}{\partial \xi^{2}}+\frac{i k}{\epsilon^{2}}\left[\mu\left(A A_{0}+A^{*} A_{2}\right)+\nu|A|^{2} A\right]+ \\
+i k\left[\mu A_{2}^{*} A_{3}+\nu\left(A_{0}^{2} A+A^{* 2} A_{3}+2 A_{0} A^{*} A_{2}+2 A\left|A_{2}\right|^{2}\right)\right]=0
\end{gathered}
$$

where the amplitudes of the zero, second and third harmonics can be found from the counterparts of equations (6) and (8)

$$
\begin{gathered}
3 \lambda k^{2} \frac{\partial A_{0}}{\partial \xi}+\epsilon^{2} \frac{\partial A_{0}}{\partial \tau}+ \\
+\frac{\partial}{\partial \xi}\left\{\mu|A|^{2}+\epsilon^{2}\left[\frac{\mu}{2}\left(A_{0}^{2}+2\left|A_{2}\right|^{2}\right)+\nu\left(2 A_{0}|A|^{2}+A^{* 2} A_{2}+A^{2} A_{2}^{*}\right)\right]\right\}=0 \\
-i k\left(6 \lambda k^{2} A_{2}-\mu A^{2}\right)+\epsilon^{2} \frac{\partial}{\partial \xi}\left[-9 \lambda k^{2} A_{2}+\frac{\mu}{2} A^{2}\right]+ \\
+2 i k \epsilon^{2}\left[\mu\left(A_{0} A_{2}+A^{*} A_{3}\right)+\nu\left(A_{0} A^{2}+2|A|^{2} A_{2}\right)\right]=0 \\
-8 \lambda k^{2} A_{3}+\mu A A_{2}+\frac{\nu}{3} A^{3}=0
\end{gathered}
$$

Using the condition that in the transition zone $\nu \approx \mu^{2} / 6 \lambda k^{2}$ with an accuracy of $\epsilon^{2}$, the solutions of the last three equations have the following form

$$
\begin{gathered}
A_{3}=\frac{\nu^{2}}{\mu^{2}} A^{3} \\
A_{2}=\frac{\mu}{6 \lambda k^{2}} A^{2}-\epsilon^{2}\left[2 \frac{\nu^{3}}{\mu^{3}} A^{2}|A|^{2}+\frac{\nu}{i k \mu} \frac{\partial}{\partial \xi} A^{2}\right] \\
A_{0}=-\frac{\mu}{3 \lambda k^{2}}|A|^{2}+\epsilon^{2}\left(-\frac{2 \nu^{3}}{\mu^{3}}|A|^{4}+\hat{A}_{0}\right)
\end{gathered}
$$

where

$$
\frac{\partial \hat{A}_{0}}{\partial \xi}=\frac{4 \nu^{2}}{\mu^{3}} \frac{\partial}{\partial \tau}|A|^{2}
$$

As a result, equation (21) becomes

$$
\frac{\partial A}{\partial \tau}+3 i k \lambda \frac{\partial^{2} A}{\partial \xi^{2}}+i k \delta|A|^{2} A-\nu A^{*} \frac{\partial}{\partial \xi} A^{2}+i k \mu A \hat{A}_{0}=0
$$


Calculating $\partial|A|^{2} / \partial \tau$ from (29) and substituting into (28), we find that

$$
\hat{A}_{0}=\frac{4 \nu^{3}}{\mu^{3}}|A|^{4}-\frac{12 i k \lambda \nu^{2}}{\mu^{3}}\left(A^{*} \frac{\partial A}{\partial \xi}-A \frac{\partial A^{*}}{\partial \xi}\right) .
$$

As a result, the modified nonlinear Schrodinger equation becomes

$$
\frac{\partial A}{\partial \tau}+3 i \lambda k \frac{\partial^{2} A}{\partial \xi^{2}}+i k \delta|A|^{2} A+4 i k \frac{\nu^{3}}{\mu^{2}}|A|^{4} A-2 \nu A^{2} \frac{\partial A^{*}}{\partial \xi}=0 .
$$

It is convenient to transform (31) to

$$
i \frac{\partial A}{\partial \eta}=\frac{\partial^{2} A}{\partial \xi^{2}}+\alpha|A|^{2} A+\beta|A|^{4} A+i \gamma A^{2} \frac{\partial A^{*}}{\partial \xi} .
$$

using the following transformation

$$
\eta=3 \lambda k \tau, \quad \alpha=\frac{\delta}{3 \lambda}, \quad \beta=\frac{4 \nu^{3}}{3 \lambda \mu^{2}}, \quad \gamma=\frac{2 \nu}{3 \lambda k}
$$

Equations of the form (32) have been derived in several physical context (e.g. [12]).

The two additional two nonlinear terms in the modified nonlinear Schrodinger equation change the dynamics of large-amplitude wave groups. In particular, presenting the complex wave amplitude in the form $A=\left(B_{0}+b\right) \exp \left(i\left(\Phi_{0}+\Phi\right)\right.$, where $\Phi_{0}=\left(-\alpha B_{0}^{2}+\beta B_{0}^{4}\right) t$, we obtain the dispersion relation for a weak perturbation of the nonlinear plane wave $(b, \Phi \sim \exp (i(\Omega t-K x))$

$$
\Omega^{2}=-\left(2 \alpha B_{0}^{2}+4 \beta B_{0}^{4}-\gamma^{2} B_{0}^{4}\right) K^{2}+K^{4} .
$$

Modulational instability occurs if

$$
2 \alpha+\left(4 \beta-\gamma^{2}\right) B_{0}^{2}>0
$$

Here $\left(4 \beta-\gamma^{2}\right)>0$ (see 33$)$ and therefore modulational instability can only occur for $\alpha>0$ (i.e. $\delta>0$ ) for any amplitude. But if $\alpha<0(\delta<0)$ modulational instability can only occur if the wave amplitude exceeds the critical value

$$
|A|>A_{c r}=\sqrt{\frac{2|\alpha|}{4 \beta-\gamma^{2}}}=\sqrt{\frac{|\delta| \mu^{2}}{4 \nu^{3}}} .
$$

Soliton solutions of equation (32) can be found analytically. The complex function $A$ should be presented in form

$$
A(\xi, \eta)=B(\xi) \exp i\left[\Theta(\xi)-Q^{2} \eta\right]
$$

After substitution in (32), we readily obtain the following relation between the amplitude and phase,

$$
\Theta=-\frac{\gamma}{4} \int B^{2} d \xi
$$


while the amplitude $B$ is given by the ordinary differential equation

$$
\frac{d^{2} B}{d \xi^{2}}-Q^{2} B+\alpha B^{3}+\chi B^{5}=0,
$$

where

$$
\chi=\beta-\frac{5}{16} \gamma^{2}=\frac{\nu^{3}}{2 \lambda \mu^{2}}>0 .
$$

The solution of (39) gives the soliton,

$$
B=\frac{2 Q}{\sqrt{\alpha+\sqrt{\alpha^{2}+\frac{16 \chi Q^{2}}{3} \cosh (2 Q \xi)}}}
$$

If $\alpha>0$ the soliton amplitude varies from zero to infinity. In the small-amplitude limit, the expression (41) transforms to the envelope soliton (17), and for large amplitudes the soliton reduces to

$$
B=\sqrt{\frac{\sqrt{3 / \chi} Q}{\cosh (2 Q \xi)}}
$$

The same expression is obtained exactly when $\alpha=0$ (the boundary of the Benjamin-Feir instability domain). The energy (momentum) of this soliton does not depend on the wave amplitude. Usually, such solutions are sensitive to external perturbation terms to (31) and structurally unstable [13], but this analysis is beyond the scope of this paper.

For $\alpha<0$ (outside of the Benjamin-Feir instability zone) the soliton exists only if its amplitude exceeds the critical value

$$
B_{\min }=\sqrt{\frac{3|\alpha|}{2 \chi}}=\sqrt{\frac{|\delta| \mu^{2}}{\nu^{3}}} .
$$

This condition can be expressed through $K=k-k_{c r}\left(k_{c r}=\mu^{2} / 6 \lambda \nu\right)$

$$
B_{\min }=\frac{\mu}{\nu} \sqrt{\frac{2|K|}{k_{c r}}} .
$$

It is important to note that this minimal soliton amplitude exceeds twice the critical value for the Benjamin-Feir instability. In this limit the soliton transforms into the algebraic form

$$
B=\sqrt{\frac{6|\alpha|}{4 \chi+3 \alpha^{2} \xi^{2}}}
$$

For large amplitudes the soliton is again given by (42). When $|\alpha|$ is large, the soliton amplitude is also large and the weak-amplitude approximation falls. 


\section{The breather solution of the extended Korteweg- de Vries equation}

The asymptotic analysis of wave group dynamics in the weakly nonlinear limit described in the previous two sections can be compared with exact solutions of the extended Korteweg-de Vries equation (1) representing nonlinear wave groups (i.e. breathers). In the framework of the canonical modified Korteweg-de Vries equation it has been shown that breathers exist only for a positive sign of the coefficient $\nu$ of the cubic nonlinear term. Taking into account that the extended Korteweg-de Vries equation can be reduced to the modified Korteweg-de Vries equation, breathers should also exist only for $\nu>0$. The expected solutions for breathers in the framework of (1) were obtained by Pelinovsky \& Grimshaw [14] for this case and for any value of the coefficient $\mu$ of the quadratic nonlinear term. It has the following form (for simplicity, $\lambda=1, \mu=12 q, \nu=6$, where $q$ is arbitrary),

$$
u=2 \frac{\partial}{\partial x} \tan ^{-1} \frac{l \cosh (\Psi) \cos (\theta)-k \cos (\Phi) \sinh (\kappa)}{l \sinh (\Psi) \sin (\theta)+k \sin (\Phi) \cosh (\kappa)}
$$

where $\theta$ and $\kappa$ are "travelling" phases,

$$
\theta=k(x-w t)+\theta_{0}, \quad \kappa=l(x-v t)+\kappa_{0},
$$

propagating with the velocities,

$$
w=-k^{2}+3 l^{2}, \quad v=-3 k^{2}+l^{2} .
$$

This solution has four free parameters, two initial phases $\left(\theta_{0}\right.$ and $\left.\kappa_{0}\right)$ and two "energetic" parameters:

$$
\Phi+i \Psi=\tan ^{-1}\left[\frac{l+i k}{2 q}\right] .
$$

It is convenient to express the "local" wavenumbers, $k$ and $l$, through these parameters, $\Phi$ and $\Psi$,

$$
\begin{aligned}
& k=q \frac{\sinh (2 \Psi)}{\cos ^{2}(\Phi) \cosh ^{2}(\Psi)+\sin ^{2}(\Phi) \sinh ^{2}(\Psi)}, \\
& l=q \frac{\sin (2 \Phi)}{\cos ^{2}(\Phi) \cosh ^{2}(\Psi)+\sin ^{2}(\Phi) \sinh ^{2}(\Psi)} .
\end{aligned}
$$

The relationship between the wavenumbers of the carrier and envelope waves can vary widely. We investigate here only slowly modulated wave groups which can be obtained from this breather solution when $l<<k$. This limit follows when $\Phi \rightarrow \pi / 2$, and $\Psi$ is fixed. In this case $w$ and $v$ are the linear phase and group velocities, $c_{p}$ and $c_{g}$ respectively, we can omit the term with $l$ in the 
dominator of (46) and replace $\tan ^{-1}(z)$ with $z$. As a result, the expression (46) reduces to

$$
u=\frac{2 l}{\sqrt{1-4 q^{2} / k^{2}}} \operatorname{sech}\left[l\left(x-c_{g} t\right)\right] \sin \left[k\left(x-c_{p} t\right)\right],
$$

which coincides with (17) on taking into account the values of the coefficients. Also, from (50) it follows that

$$
k=2 q \operatorname{coth} \Psi
$$

and, therefore $k>2 q$, as it should be from the criterion (13) for modulational instability. It is important to mention that in the vicinity of the boundary of the modulational instability domain, the breather amplitude grows indefinitely $(\Psi \rightarrow \infty)$, and our approximation is not valid.

Thus, the weakly nonlinear breather is an envelope soliton, and its parameters satisfy the modulational instability criterion. If the coefficient of the quadratic nonlinear term in the extended Korteweg-de Vries equation is zero, the wavenumber of a weakly nonlinear breather can have any value according to the modulational instability criterion (13) of the modified Korteweg-de Vries equation.

Another limit for a slowly modulated wave packet can be obtained from the breather solution when $\Psi \rightarrow \infty$ for fixed $0<\Phi<\pi / 2$. In this case $k \simeq 2 q$ and this corresponds to the boundary of the modulational instability domain and the consequent applicability of the modified nonlinear Schrodinger equation. Introducing the deviation $K$ from this boundary by $k=2 q+K,|K|<<2 q$, from (50) and (51) we get

$$
\begin{gathered}
K=-4 q \cos (2 \Phi) \exp (-2 \Psi), \\
l=4 q \sin (2 \Phi) \exp (-2 \Psi),
\end{gathered}
$$

and

$$
\tan (2 \Phi)=-\frac{l}{K}
$$

In particular, for positive $K$ (when the cooefficient $\delta$ of the cubic nonlinear term in the nonlinear Schrodinger equation is positive) we have $\Phi>\pi / 4$, and for negative $K(\delta<0) \Phi<\pi / 4$. If $\Phi$ is not close to zero, both terms with $l$ in (46) are small and with accuracy $l / k$ the solution (46) becomes

$$
u=\frac{2 l}{1+\cot ^{2}(\Phi) \tanh ^{2}(\kappa)}\left[\frac{-\cosh (\Psi) \sin (\theta)+\sinh (\Psi) \cot (\Phi) \tanh (\kappa) \cos (\theta)}{\sin (\Phi) \cosh (\kappa)}\right]
$$

Taking into account $\Psi \rightarrow \infty$, this transforms to

$$
u=\frac{l \exp (\Psi)}{\sin (\Phi) \cosh (\kappa)\left[1+\cot ^{2}(\Phi) \tanh ^{2}(\kappa)\right]}[-\sin (\theta)+\cot (\Phi) \tanh (\kappa) \cos (\theta)]
$$


Rewriting it in the form of (3), the complex amplitude of the first harmonic can be obtained as

$$
\left.A=\frac{l \exp (\Psi)}{\sqrt{2\left[\cosh ^{2}(\kappa)-\cos ^{2}(\Phi)\right]}} \exp -i \Theta\right)
$$

where

$$
\cot (\Theta)=\cot (\Phi) \tanh (\kappa) .
$$

It is convenient to write the modulus of wave amplitude in the form of (41)

$$
B=\sqrt{\frac{l^{2} \exp (2 \Psi)}{2[\cosh (2 l \xi)-\cos (2 \Phi)]}} .
$$

After substitution of $\Phi$ and $\Psi$ from (54) and (55), the final version is

$$
B=\sqrt{\frac{2 q l^{2}}{K+\sqrt{K^{2}+l^{2}} \cosh (2 l \xi)}},
$$

which fully coincides with (41).

When $l \rightarrow 0$, or $\Phi \rightarrow 0$, we should substitute (55) and( 54) into (46) and take the limit $\rightarrow 0$,

$$
u=-2 \frac{\partial}{\partial x} \tan ^{-1}\left[\sqrt{\frac{|K|}{2 q}} \frac{\cos (\theta)-2 q \sqrt{2|K| / q}(x-v t)}{1+\sqrt{|K| / 2 q} \sin (\theta)}\right] .
$$

This "algebraic" breather has an envelope in the form (45) of the algebraic soliton of the modified nonlinear Schrodinger equation.

Thus, we have full agreeement between solutions of the modified Schrodinger equation and the weakly nonlinear breather solution of the extended Kortewegde Vries equation. For large negative $K$ the carrier wavenumber of the breather decreases, its amplitude increases and the breather represents solitons of opposite polarity. Because solitons of different polarity have different amplitudes (for the same value of the propagation speed), and the "negative" soliton should exceeds the amplitude of the algebraic soliton $(2 \mu / \nu)$, such a breather is a strongly nonlinear wave.

\section{Numerical simulations}

Our aim here is to compute the evolution of wave packets within the framework of the various models described in the previous sections. The extended Korteweg-de Vries (eKdV) equation is solved using a finite-difference scheme, while the nonlinear Schrodinger equation (NLS) is solved with a pseudo-spectral method. The boundary conditions for both equations are periodic. The length of the domain is 400 , the carrier wavenumber is $k=1.0048$ (60 wavelengths in domain). The coefficient of the dispersion term in the extended Korteweg-de Vries equation is set as $\lambda=1$, the coefficient of the quadratic nonlinear term is 
set as $\mu=4$, while the coefficient $\nu$ of the cubic nonlinear term is varied from 5 to -1 , providing both modulated and demodulated regimes (the coefficient of the cubic nonlinear term in the nonlinear Schrodinger equation is such that $\delta=0$ for $\nu_{c r}=2.641$ ). The initial condition for the extended Korteweg-de Vries equation is taken in the form

$$
u(x, 0)=A_{0} \exp \left(-K^{2} x^{2}\right) \sin (k x),
$$

and for the nonlinear Schrodinger equation is

$$
A(x)=\frac{A_{0}}{2} \exp \left(-K^{2} x^{2}\right)
$$

where $A_{0}=0.2$ and $K=0.0157$.

The first simulation is for $\nu=5$ and corresponds to a zone of modulational instability. Our simulations are presented in Fig. 1 for both models, the extended Korteweg-de Vries equation (left) and the nonlinear Schrodinger equation (right); note that for the latter case we restore the carrier wave to better facilitate the comparison. First, we should point out that equation (1) is solved in fixed coordinates, and a wave packet propagates to the left (in the linear case with the group velocity, $c_{g r}=-3 k^{2}$ ), but equation (10) is written for a system of coordinates propagating with this linear group velocity. The dynamics of the wave packet in the framework of the nonlinear Schrodinger equation follows known scenarios: an initial disturbance transforms into envelope solitons and dispersive tails. The number of solitons can be found from the corresponding eigenvalue problem (which is written for the "classical" form (15) of the nonlinear Schrodinger equation)

$$
\begin{aligned}
& \frac{d F_{1}}{d x}=\varpi F_{1}-A_{1}(x) F_{2} \\
& \frac{d F_{2}}{d x}=\varpi F_{2}+A_{1}(x) F_{1}
\end{aligned}
$$

where $A_{1}=\sqrt{\delta / 6} A$ (see (14)) and $\varpi$ is the complex eigenvalue, in general. When the initial disturbance (65) has a single polarity, it is known [11] that all discrete eigenvalues are real and determine the soliton amplitudes as $A_{\text {sol }}=$ $4 \sqrt{6 / \delta} \varpi$. Here the discrete levels $\varpi$ are 0.05 and 0.026 . As a result, two envelope solitons should form with amplitudes 0.33 and 0.16 . Because the group velocities are the same, these solitons cannot split in space, and so interact between themselves. Figure 1 demonstrates the transformation of an initial Gaussian impulse (65). It is interesting to compare the wave groups during this process in both models. At the time moment $t=40$ an asymmetry for the eKdV (left) is evident and a small-amplitude second harmonic group leaves the initial group. There is full symmetry for the NLS (right) The first occurence of an envelope soliton is at $t=100$ for both models and corresponding plots are very similar. As mentioned above, two envelope solitons should form, but at $t=100,140$ they have not yet separated, and we see similar large impulses 
and two adjointing wave groups in both plots. At $t=400$ two solitons and a small dispersive tail appear in the eKdV model (left), and a large impulse with two adjointing small wave groups, and a small symmetric tail are seen in the right-hand side plot ((NLS). After this time, the left-hand side and righthand plots are not similar. The NLS model demonstrates a periodic process of interaction of two solitons with recurrence at $t=600$. The eKdV model shows a complex interaction and separation of both solitons. The amplitudes of the envelope solitons are in good agreement with predicted values based on the inverse scattering method.

The next simulation is for $\nu=-1$. This case corresponds to the demodulated nonlinear Schrodinger equation $(\delta<0)$. Simulations of a Gaussian wave packet are presented in Fig. 2 (left for the eKdV equation and right for the NLS equation). As expected, the wave packet disperses, its amplitude decreases, and its length increases. The difference between the two models is related to the generation of free wave groups for the second and "zero" harmonics as mentioned above. Similar results are obtained also for $\nu=0$ (Korteweg-de Vries equation), see $[19],[17]$.

The modelling of the wave group evolution in the transition zone $(\delta \approx 0)$ presents some technical difficulties due to an increase in the characteristic spatial and temporal scales, that requires a more accurate numerical scheme. The length of domain is increased to 1000 , the cubic nonlinear coefficient is set at $\nu=6$, the quadratic nonlinear coefficient is set at $\mu=3$. For these parameters, the critical value of the carrier wavenumber is $k_{c r}=0.5$. First, we checked the stability and steady-state (for the envelope) propagation of the breather (46), see Fig. 3 for $K>0(\Phi=0.8, \Psi=2)$. It is clear from Fig. 3 that no tail is formed (compare the simulations in Fig.1), because the breather is an exact solution of the $\mathrm{KdV}$ equation. Then, this breather solution is multiplied by 1.05 and then used as the initial condition for (1). Results of these simulations are presented in Fig. 4. As expected, the wave "undresses" its tail and transforms to a new breather state. In the transition zone, the wave envelope is not symmetric. The same features occur for the "reduced" breather, where amplitude is $0.9 u_{0}(x)$, see Fig. 5. When $K>0$ the breather is stable, and if an initial disturbance is greater than the breather, it transforms back to the breather with a dispersive tail, similar to Fig. 4. More interesting is the case, when the initial amplitude is less than the minimal amplitude of the breather ("algebraic" breather), see (63) and (43). In our case $B_{\min }=0.126$ (for $\Phi=0.25, \Psi=2, K=-0.016$ ). The evolution of this small breather-like initial disturbance is shown in Fig. 6, and demonstrates the demodulation of the wave packet and its dispersion.

The last series of simulations were for the periodic sine modulation,

$$
A(x)=A_{0}[1+0.1 \sin K x] \sin k x .
$$

Results for the case $\mu=4, \nu=2, \lambda=1, k=1.57\left(k_{c r}=4 / 3\right), K=0.0157$, $A_{0}=0.2$ are presented in Fig. 7 for the $\mathrm{KdV}$ equation and the NLS equation. Snapshots show the development of the Benjamin-Feir instability with formation of solitons and breathers, and their recurrence. Again here the simulation in the 
framework of the $\mathrm{eKdV}$ equation shows more asymmetric forms for the wave envelopes. The NLS equation describes the wave evolution correctly only at the beginning stage.

\section{Conclusions}

These results show the link between the solutions of the extended Korteweg-de Vries equation and the nonlinear Schrodinger equation for the description of the wave groups. The dynamics of solitons and breathers in the extended Kortewegde Vries model is determined by the sign of the coefficient $\nu$ of the cubic nonlinear term. Correspondingly, the dynamics of wave groups in the weakly nonlinear approximation is determined by the sign of the coefficient of its cubic nonlinear term. This parameter of the nonlinear Schrodinger equation is calculated for the extended Korteweg-de Vries equation, and the corresponding nonlinear Schrodinger equation is derived. It is shown that weakly nonlinear wave groups with small carrier wavenumbers are always stable, but high wavenumber wave groups are always unstable and form wave packets (i.e. envelope solitons). For the transition zone where the coefficient $\delta \approx 0$, a modified nonlinear Schrodinger equation is derived, and its steady-state solitary wave solutions are found. In particular, it is shown that this equation has a soliton of algebraic form. The exact breather solution of the extended Korteweg-de Vries equation is investigated in the weakly nonlinear limit. It is shown that the breather coincides with solutions of the nonlinear Schrodinger equation and its modification in the transition zone. Our numerical simulations demonstrate the main features of the wave dynamics for a wide range of parameter, exhibiting modulation and demodulation, and the formation of envelope solitons and breathers. For the same conditions the solutions of the extended Korteweg-de Vries equation have a more asymmetric form than that predicted in the framework of the nonlinear Schrodinger equation. Also, the steady-state wave packets of the extended Korteweg-de Vries equation move relative to each other, a feature not seen in the nonlinear Schrodinger equation.

\section{Acknowledgement}

This work was done with financial support from a Large ARC grant, and (for EP) INTAS (99-1068), (for TT) INTAS 99-1637 and RFBR 00-05-64223.

\section{References}

[1] Ablowitz, M.J., and Segur H. Solitons and the inverse scattering transform. SIAM, Philadelphia. 1981.

[2] Akhmediev, N.N., Eleonskii, V.M., and Kulagin, N.E., Generation of periodic trains of picosecond pulses in an optical fiber: exact solutions. Sov. Phys. JETP, 1985, v. 62, 894-899 (1985). 
[3] Clarke, S., Grimshaw, R., Miller, P., Pelinovsky, E., and Talipova, T. On the generation of solitons and breathers in the modified Korteweg-de Vries equation. Chaos, 2000, v. 10, N. 2, 383 - 392 .

[4] Dysthe, K.B., and Trulsen, K., Note on breather type solutions of the NLS as a model for freak-waves. Physica Scripta, 1999, v. T82, 48-52.

[5] Grimshaw, R. Internal solitary waves. Environmental stratified flows. Kluwer. 2000.

[6] Grimshaw, R., Pelinovsky, E., and Talipova, T. The modified Korteweg-de Vries equation in the theory of large-amplitude internal waves. Nonlinear Processes in Geophysics, 1997, v. 4, N. 4, 237 - 350.

[7] Grimshaw, R., Pelinovsky, E., and Talipova, T. Solitary wave transformation in a medium with sign-variable quadratic nonlinearity and cubic nonlinearity. Physica D, 1999, v. 132, $40-62$.

[8] Henderson, K.L., Peregrine, D.H., and Dold, J.W., Unsteady water wave modulations: fully nonlinear solutions and comparison with the nonlinear Schrodinger equation. Wave Motion, 1999, v. 29, 341-361.

[9] Kakutani, T., and Yamasaki, N. Solitary waves on a two-layer fluid. J. Phys. Soc. Japan, 1978, v. 45, $674-679$.

[10] Ma, Y.-Ch., The perturbed plane-wave solutions of the cubic Schrodinger equation. Stud. Appl. Math., 1979, v. 60, 43-58.

[11] Newell, A.C. Solitons in mathematics and physics. SIAM, Philadelphia. 1985.

[12] Parkes E.J. The modulation of weakly non-linear dispersive waves near the marginal state of instability. J. Phys. A: Math. Gen. 1987, v. 20, 2025 2036 .

[13] Pelinovsky, D. Radiative effects to the adiabatic dynamics of envelope-wave solitons. Physica D., 1998, v. 119, $301-313$.

[14] Pelinovsky, D., and Grimshaw, R. Structural transformation of eigenvalues for a perturbed algebraic soliton potential. Physics Letters A, 1997, v. 229, $165-172$.

[15] Pelinovsky, E., Talipova, T., and Kharif, C. Nonlinear dispersive mechanism of the freak wave formation in shallow water. Physica D, 2000, v. 147, 83-94.

[16] Peregrine, D.H., Water waves, nonlinear Schrodinger equations and their solutions. J. Austral. Math. Soc. Ser. B, 1983, v. 25, 16-43. 
[17] Kit, E., Shemer, L., Pelinovsky, E., Talipova, T., Eitan, O., and Jiao, H. Nonlinear wave group evolution in shallow water. J. Waterway, Port, Costal, Ocean Eng., 2000, v. 126, N. 5, 221 - 228.

[18] Slyunyaev, A., and Pelinovsky, E. Dynamics of large-amplitude solitons. JETP, 1999, v. 89, N. 1, $173-181$.

[19] Talipova, T., Pelinovsky, E., Kit, E., and Eitan, O. Nonlinear transformation of wave packets in weakly dispersive media. Radiophysics and Quantum Electronics, 1999, v. 42, N. 4, $315-319$.

[20] Zakharov, V.E., and Kuznetsov, E.A. Multi-scale expansions in the theory of systems integrable by the inverse scattering transform. Physica D., 1986, v. $18,455-463$. 


\section{Figure Captions}

Fig. 1. Simulation of wave packet in the regime of modulational instability (left - extended Korteweg-de Vries equation, right - nonlinear Schrodinger equation) Fig. 2. Simulation of wave packet in the regime of modulational stability (left - extended Korteweg-de Vries equation, right - nonlinear Schrodinger equation) Fig 3. Steady-state propagation of a breather, $u_{0}(x)\left(k=0.5002>k_{c r}=0.5\right)$

Fig. 4. Evolution of the initial disturbance, $1.05 u_{0}(x)$ for $k=0.5002>k_{c r}=0.5$ Fig. 5. Evolution of the initial disturbance, $0.9 u_{0}(x)$ for $k=0.5002>k_{c r}=0.5$ Fig. 6. Evolution of an initial disturbance with an amplitude less than the minimal value for $k=0.484<k_{c r}=0.5$

Fig. 7. Snapshot of the periodically modulated wavetrain (left - the extended Korteweg- de Vries equation, right - the nonlinear Schrodinger equation). 

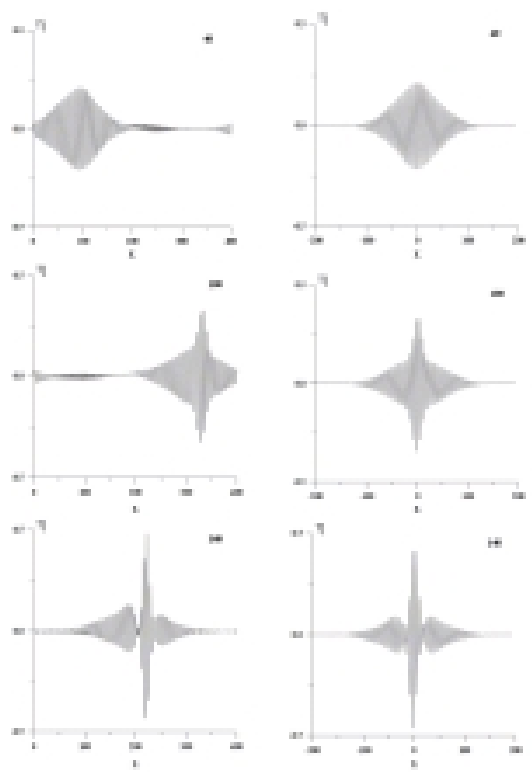

Fig 1. Simulation of wave packet in the regime of modulational instability (left extended Korteweg-de Vries equation, right - nonlinear Schrodinger equation). 

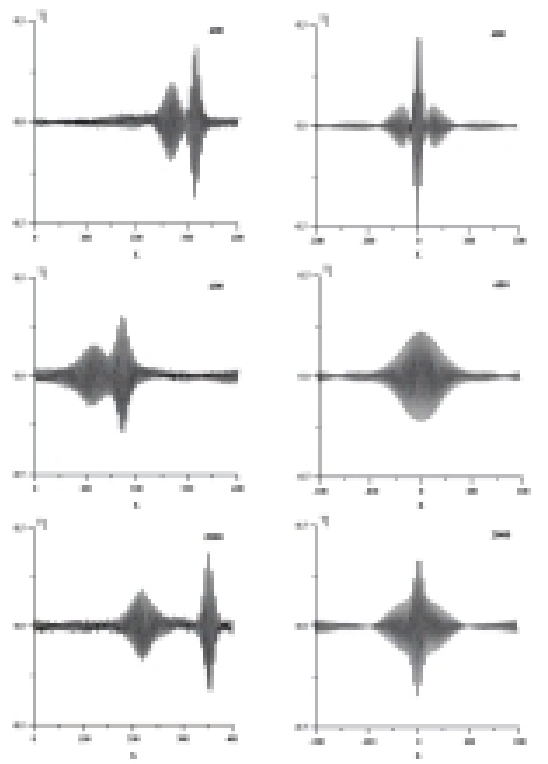

Fig 1. Simulation of wave packet in the regime of modulational instability (left extended Korteweg-de Vries equation, right - nonlinear Schrodinger equation). (continued) 


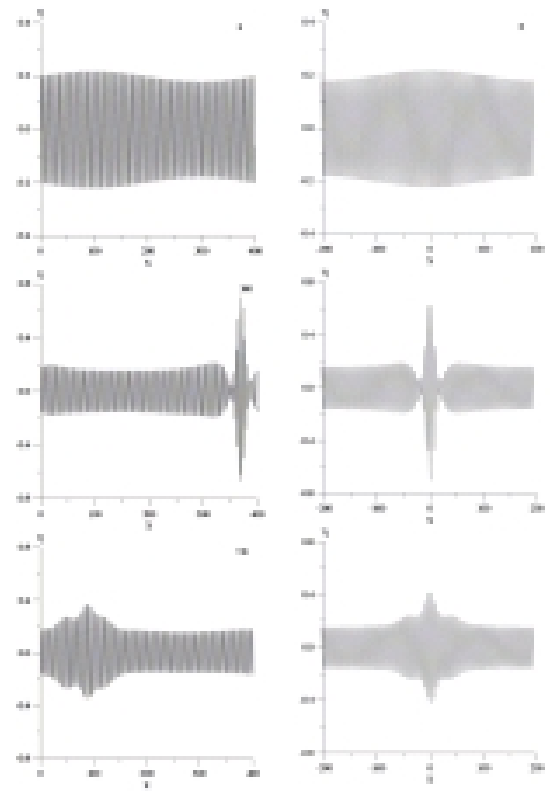

Fig. 7. Snapshot of the periodically modulated wavetrain (left - the extended Korteweg- de Vries equation, right - the nonlinear Schrodinger equation). 


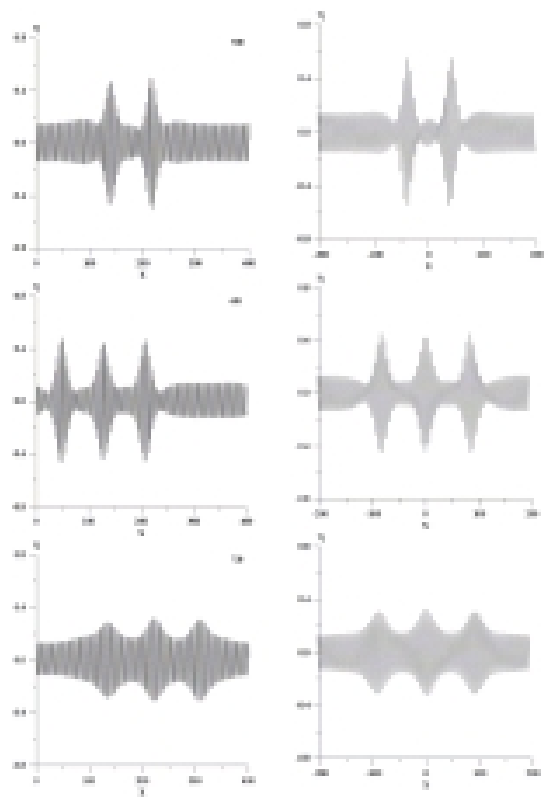

Fig. 7. Snapshot of the periodically modulated wavetrain (left - the extended Korteweg- de Vries equation, right - the nonlinear Schrodinger equation). (continued)

Figure 1: 


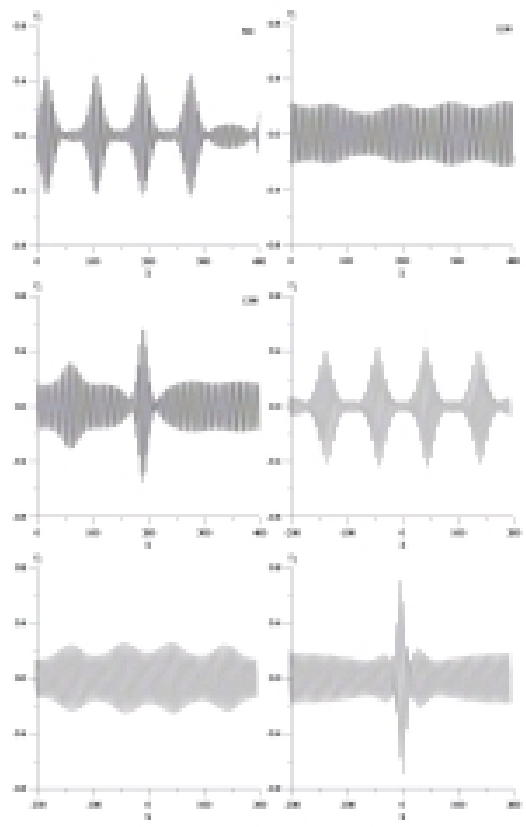

Fig. 7. Snapshot of the periodically modulated wavetrain (left - the extended Korteweg- de Vries equation, right - the nonlinear Schrodinger equation). (continued) 